\title{
Cardiovascular Applications of Hyperpolarized MRI
}

\author{
Damian J. Tyler
}

Published online: 19 January 2011

(C) The Author(s) 2011. This article is published with open access at SpringerLink.com

\begin{abstract}
Many applications of MRI are limited by an inherently low sensitivity. Previous attempts to overcome this insensitivity have focused on the use of MRI systems with stronger magnetic fields. However, the gains that can be achieved in this way are relatively small and increasing the magnetic field invariably leads to greater technical challenges. More recently, the development of a range of techniques, which can be gathered under the umbrella term of "hyperpolarization," has offered potential solutions to the low sensitivity. Hyperpolarization techniques have been demonstrated to temporarily increase the signal available in an MRI experiment by as much as 100,000-fold. This article outlines the main hyperpolarization techniques that have been proposed and explains how they can increase MRI signals. With particular emphasis on the emerging technique of dynamic nuclear polarization, the existing preclinical cardiovascular applications are reviewed and the potential for clinical translation is discussed.
\end{abstract}

Keywords Hyperpolarization · Dynamic nuclear polarization · Parahydrogen-induced polarization $\cdot$ Optical pumping $\cdot$ Brute force polarization - Magnetic resonance imaging $\cdot$ Magnetic resonance spectroscopy $\cdot$ Cardiac metabolism · Angiography $\cdot$ Perfusion $\cdot$ Catheter tracking

D. J. Tyler $(\bowtie)$

Cardiac Metabolism Research Group, Department of Physiology,

Anatomy \& Genetics, University of Oxford,

Sherrington Building, Parks Road,

Oxford, UK OX1 3PT

e-mail: damian.tyler@dpag.ox.ac.uk

\section{Introduction}

In recent years the development of cardiac magnetic resonance imaging (CMR) has provided a valuable new approach for the assessment of cardiac structure and function. However, despite the enormous developments in terms of hardware, software, and processing power that have taken place, MRI remains an inherently sensitivity limited technique and certain applications are restricted by the low signal levels, e.g. multi-nuclear spectroscopy for the assessment of metabolism [1]. The low sensitivity is due to the fact that the interactions between the static magnetic field of the MRI system and the molecules of the body are small. The standard approach to overcome this sensitivity limitation has been to increase the magnetic field strength of the MRI system, such that MRI systems with static field strengths of $3 \mathrm{~T}$ are now becoming commonplace for clinical practice and ultra-high field strength systems at $7 \mathrm{~T}$ or above are being used in the research setting. However, the gains in signal that can be achieved by increasing field strength are limited because the signalto-noise ratio (SNR) scales approximately linearly with the magnetic field strength. Added to this limited increase in sensitivity are the increasing technical and safety challenges that are faced at higher field strengths in terms of $B_{1}$ field homogeneity, specific absorption rate (SAR) levels, system costs, etc.

An alternative approach to the issue of low sensitivity is offered by a range of techniques that are commonly described under the envelope of "hyperpolarization" [25]. These techniques work by temporarily increasing the strength of the MR signal, in some cases by as much as 100,000-fold [2, 6]. All hyperpolarization techniques require the injection, infusion, or inhalation of a substance that has been previously prepared in a separate system and 
the large increases in sensitivity have been utilized in a wide range of different applications, including lung imaging [4], tumor staging [7, 8], and the assessment of cardiovascular diseases $[9 \bullet \bullet, 10 \bullet, 11 \bullet \cdot$. All hyperpolarized techniques also suffer from the same inherent problem; the increased sensitivity is transient, and so the decay of the increased signal must be carefully controlled within the experiment [1].

The aim of this review is to describe the main types of hyperpolarization techniques that are available and, with particular focus on the emerging technique of dynamic nuclear polarization (DNP), to describe the existing literature on the preclinical application of hyperpolarized agents to study the heart. The review will then close with a discussion of the potential for translation of the DNP technique into humans and the main challenges that need to be overcome.

\section{Hyperpolarization Techniques}

The basis of magnetic resonance imaging lies in the interaction between the static magnetic field of the MRI system and the molecules of the body. When placed in the magnetic field, the molecules act like small bar magnets, aligning themselves in one of two orientations, either in the same direction as the field or opposed to it. The signal generated by the MRI system is then proportional to the difference in the number of molecules aligned in the two orientations, referred to as the polarization. At normal clinical magnetic field strengths and room temperature, the polarization is very small (e.g. at $3 \mathrm{~T}$, the difference between the number of molecules in the different orientations is only 10 molecules in every million). The aim of hyperpolarization techniques is to artificially increase the polarization, and thus the signal from the MRI system, by forcing more of the molecules into one orientation. Currently, there are four main approaches used to generate hyperpolarized compounds, which can subsequently act as high-sensitivity tracers in an MRI experiment. These are brute force polarization [5], optical pumping of noble gases [4], parahydrogen-induced polarization (PHIP) [2], and dynamic nuclear polarization (DNP) $[3,6]$.

\section{Brute Force Polarization}

The brute force approach is perhaps the simplest to understand but currently the least effective. It involves placing the sample to be polarized in a very high magnetic field at extremely low temperatures. In these conditions the sample will naturally approach a high level of polarization because the polarization achieved by a sample increases with the magnitude of the static magnetic field and decreases with the temperature. However the brute force approach is limited because the size of the polarization levels that can be achieved are relatively small and the sample can take a long time to approach the high polarization level. There are also practical difficulties in returning the sample to the liquid state for use in an in vivo experiment [5].

\section{Optical Pumping}

Optical pumping of the noble gases, particularly helium $\left({ }^{3} \mathrm{He}\right)$ and xenon $\left({ }^{129} \mathrm{Xe}\right)$, has generated a great deal of interest in recent years due to its direct applicability to the imaging of the lungs [4]. The sample to be polarized is mixed with either an alkali-metal vapor or a vapor of metastable atoms. The mixture is then irradiated with circularly polarized light from a laser source, which is absorbed by the alkali or metastable atoms, increasing the polarization of their electrons [5]. This high level of polarization is then passed on to the nuclei of the noble gas through collisions. MR images of the lungs generated following the inhalation of these hyperpolarized noble gases can then be used to detect changes in ventilation, perfusion, and lung microstructure associated with diseases such as chronic obstructive pulmonary disease (COPD) and asthma [4].

\section{Parahydrogen-Induced Polarization}

PHIP, also referred to as PASADENA, utilizes a highly polarized form of molecular hydrogen, known as parahydrogen, to increase the polarization of a specific nucleus, typically carbon $\left({ }^{13} \mathrm{C}\right)$ or nitrogen $\left({ }^{15} \mathrm{~N}\right)$, within a chosen molecule [2]. The first step in the PHIP technique is to generate high levels of parahydrogen. Molecular hydrogen is normally found in two different forms, orthohydrogen and parahydrogen, in a 3:1 ratio. At low temperatures, and in the presence of a suitable catalyst, the relative abundance of parahydrogen can be increased to nearly $100 \%$. The parahydrogen produced is then involved in a hydrogenation reaction, in the presence of a second catalyst, that produces the molecule to be hyperpolarized. Finally, the high polarization of the parahydrogen is transferred to the ${ }^{13} \mathrm{C}$ or ${ }^{15} \mathrm{~N}$ nuclei by either cycling the magnetic field or applying a sequence of RF pulses [5]. The PHIP technique can rapidly generate extremely high levels of polarization (> 40\%) using relatively simple and inexpensive apparatus [2]. However, the technique is limited by the specificity of the chemical reaction required and the need to remove the hydrogenation reaction catalyst before injection.

\section{Dynamic Nuclear Polarization}

The final technique, and the focus of the rest of this article, is dynamic nuclear polarization (DNP) [3, 6]. As with 
PHIP, the DNP technique is used to enhance the polarization of a specific nucleus (again typically ${ }^{13} \mathrm{C}$ or ${ }^{15} \mathrm{~N}$ ) within a particular molecule. It requires the mixing of the molecule to be hyperpolarized with a source of free electrons, known as a radical. The mixed sample is then placed in a high magnetic field (typically $3.35 \mathrm{~T}$ ) and rapidly frozen in liquid helium, reducing the sample temperature to approximately $1 \mathrm{~K}$. In these conditions, the free electrons are nearly $100 \%$ polarized (ie, all aligned in the same direction as the static magnetic field), whereas the nuclear spins of the sample are still relatively poorly polarized. The high electron polarization can then be transferred to the nuclear spins through the irradiation of the sample with microwave energy at a specific frequency, which is related to the magnetic field strength and the atomic properties of the nuclei and radical within the sample. Further details of the processes involved in this polarization transfer are more thoroughly described in the review by Comment et al. [3].

Despite the high level of polarization that can be achieved, the biological application of DNP has been limited by the need for the hyperpolarization process to take place in the solid state. This limitation was removed by the development of the dissolution DNP process [6], whereby the highly polarized solid sample is rapidly brought into the liquid state, retaining a large proportion of the enhanced polarization. The process, first described by Ardenkjaer-Larsen and colleagues in 2003, requires the rapid melting of the hyperpolarized sample with a bolus of superheated liquid and generates an injectable sample that can be used as an in vivo MR contrast agent [6].

\section{Cardiovascular Applications}

Hyperpolarization techniques have been explored in a range of cardiovascular applications. In most examples, the techniques of PHIP or DNP have been used to generate hyperpolarized ${ }^{13} \mathrm{C}$-labeled molecules that have subsequently been used for angiography, perfusion assessment, catheter tracking, or metabolic imaging [12•]. For the vascular imaging applications (angiography, perfusion, catheter tracking) the molecules used have either been non-endogenous (e.g. hydroxyethylpropionate generated by PHIP and 1,1-bis(hydroxymethyl)-cyclopropane- $\left(1-{ }^{13} \mathrm{C}\right.$, D8) generated using DNP) or endogenous molecules that were metabolically inactive (e.g. ${ }^{13} \mathrm{C}$-urea generated by DNP) [12•]. For metabolic imaging a range of different metabolically active compounds have been explored (e.g. pyruvate generated by DNP [12•] and succinate generated by PHIP [13]).
Angiography

With hyperpolarized ${ }^{13} \mathrm{C}$-labeled tracer molecules, the large SNR and the fact that there is virtually no background signal generates an extremely high contrast-to-noise ratio (CNR), which is well suited to angiographic applications [14]. As such, the use of hyperpolarized agents for angiography has been widely explored $[15,16]$, with particular focus being given to imaging of the coronary [17] and pulmonary [18] arteries. The long transverse relaxation times of ${ }^{13} \mathrm{C}$ molecules favor the use of single shot imaging techniques (e.g. trueFISP, EPI) [1] and so high temporal resolutions $(<300 \mathrm{~ms})$ have been demonstrated. The lack of background signal has also led to the use of projection imaging techniques with reasonable in-plane resolutions $(\sim 1 \mathrm{~mm})$. However, the imaging of ${ }^{13} \mathrm{C}$-labeled molecules places large demands on the imaging gradients of the MRI system because the gyromagnetic ratio of ${ }^{13} \mathrm{C}$ is $1 / 4$ that of ${ }^{1} \mathrm{H}$. This leads to the need for more powerful gradient amplifiers and, when combined with rapid imaging techniques, may place limits on the ultimate spatial resolution that is achievable for angiographic applications.

\section{Perfusion}

CMR assessments of myocardial perfusion are usually based on the first pass of a gadolinium-based contrast agent through the heart. The contrast agent shortens the $T_{1}$ of the myocardium and increases the signal intensity of well perfused tissues on $\mathrm{T}_{1}$-weighted images. However, this indirect assessment of perfusion can lead to difficulties in the absolute quantification of myocardial perfusion [19]. In contrast, hyperpolarized perfusion measurements rely directly on the signal obtained from the hyperpolarized tracer and so allow for easier absolute quantification. The continual decay of the hyperpolarized signal obviously needs to be taken into account and represents a limitation of the technique, but novel acquisition schemes and quantitative models can be developed to account for these effects [20]. The application of hyperpolarized tracers for the quantitative assessment of perfusion has been demonstrated in the pig myocardium [1] and the rat brain [21].

\section{Catheter Tracking}

The inherent lack of background signal when using hyperpolarized ${ }^{13} \mathrm{C}$ tracers also offers potential for use in interventional MR experiments. As a demonstration of this, hyperpolarized tracers have been used to enable passive catheter tracking through the aorta of a pig [22]. Through the use of a specially modified three-lumen catheter, the flowing hyperpolarized tracer was directly imaged using a 3D back-projection imaging technique that offered frame 
rates of 1.4 frames/s. The obvious limitation for hyperpolarized tracers in this application is the need to continually refresh the tracer, as the signal will decay through relaxation mechanisms and the continuous RF pulsing. However, the rapid polarization offered by the PHIP technique could provide a solution to this problem.

\section{Metabolic Spectroscopy and Imaging}

There is a growing body of evidence to suggest that alterations in metabolic substrate utilization may be a cause, rather than a symptom, of cardiovascular disease [23-25]. To increase our understanding of alterations in substrate selection, it is necessary to use an in vivo method that can repeatedly measure metabolism noninvasively. This would allow the metabolic changes which occur during disease progression to be assessed and would open a new window on the development of possible metabolic treatments. Magnetic resonance spectroscopy (MRS) using carbon $\left({ }^{13} \mathrm{C}\right)$ is ideally suited to the study of metabolism due to the extensive range of compounds that can be detected and the ability to attribute signals to the different carbon atoms within individual molecules. ${ }^{13} \mathrm{C}$ studies of cardiac metabolism have been conducted since the early 1980s when the first experiments were performed on the isolated perfused rat heart by Bailey et al. [26] using ${ }^{13} \mathrm{C}$-enriched sodium acetate. A wealth of work has since followed allowing detailed studies of metabolic substrate selection in normal [27], ischemic [28], and diabetic [29] hearts. However, the clinical utility of ${ }^{13} \mathrm{C}$ MRS experiments has been limited due to a combination of the inherently low sensitivity of MRS, the low in vivo concentrations, and the low natural abundance of ${ }^{13} \mathrm{C}$. As such, the majority of cardiac studies have been performed on ex vivo hearts, perfused with ${ }^{13} \mathrm{C}$ enriched molecules and using very long scan times. This has resulted in the ability to only study metabolic steady state conditions [30] and, so far, there have only been a limited number of in vivo studies [31] or studies of nonsteady state rates of oxidative metabolism [32] using nonhyperpolarized techniques.

The advent of hyperpolarization, particularly with DNP, has provided the ability to generate metabolically active "tracers" that can be injected into an in vivo experiment. The high SNR provided allows both the visualization of the injected molecule and also the downstream metabolic products to which the ${ }^{13} \mathrm{C}$ label is transferred. To date, the majority of experiments with this method have focused on the key metabolic molecule pyruvate, labeled with ${ }^{13} \mathrm{C}$ at the first carbon position $\left(\left[1-{ }^{13} \mathrm{C}\right]\right.$ pyruvate). Pyruvate is metabolically important because it sits at the junction of metabolism between glycolysis and the tricarboxylic acid (TCA) cycle (Fig. 1) and therefore allows a range of different metabolic pathways to be explored. $\left[1-{ }^{13} \mathrm{C}\right]$ pyruvate also offers practical advantages as it is easily hyperpolarized, is rapidly taken up into cells and metabolized, and has a long relaxation time ( $\mathrm{T}_{1}$ of $\left.\sim 60 \mathrm{~s}\right)$ meaning that the hyperpolarized signal persists for a long time.

In the first application of DNP-generated hyperpolarized $\left[1-{ }^{13} \mathrm{C}\right]$ pyruvate in the perfused rat heart, Merritt et al. [10] showed the conversion of infused pyruvate into lactate, alanine, bicarbonate, and carbon dioxide. The lactate observed has since been shown to be due to rapid exchange of the ${ }^{13} \mathrm{C}$ label with endogenous lactate via the enzyme lactate dehydrogenase (LDH) [33]. Similarly, the alanine signal is generated by the transamination reaction mediated by alanine aminotransferase (AAT). The bicarbonate resonance is observed due to the transformation of pyruvate into acetyl-CoA via the enzyme pyruvate dehydrogenase (PDH). During this enzymatic transfer, the ${ }^{13} \mathrm{C}$ label is cleaved from the pyruvate molecule as ${ }^{13} \mathrm{CO}_{2}$, which is in a rapid, pH-dependant equilibrium with bicarbonate under the action of the enzyme carbonic anhydrase (CA). Therefore, the observation of bicarbonate can be seen as a surrogate marker of flux through PDH. The ability to measure flux through PDH could be vitally important as PDH is one of the key regulatory enzymes of the glucose-fatty acid (or Randle) cycle, and is therefore a key determinant of substrate selection in the heart [34].

In the first use of hyperpolarized $\left[1-{ }^{13} \mathrm{C}\right]$ pyruvate in the in vivo rodent heart, Schroeder et al. demonstrated that not only could the metabolic fate of $\left[1-{ }^{13} \mathrm{C}\right]$ pyruvate be monitored in vivo, but that physiological (overnight fasting) and pathological (type 1 diabetes) modulation of PDH flux could be observed $[11 \bullet, 35]$. They further demonstrated that the in vivo assessment of PDH flux in their model of type 1 diabetes correlated significantly with the severity of the disease. This work was extended by Atherton et al. [36], who demonstrated that the in vivo assessment of PDH flux obtained using hyperpolarized MRS correlated significantly with the ex vivo activity of the PDH enzyme as assessed by a traditional enzymatic activity assay. In this work a period of high fat feeding was used to reduce PDH activity, and dichloroacetate (a pyruvate analogue known to increase PDH activity) was infused to generate a range of in vivo PDH activities.

Further work has since shown the applicability of the DNP technique to the study of myocardial ischemia in the perfused heart $[37,38 \bullet \bullet$, where elevated levels of lactate were observed following a period of global ischemia. Also, the ability of hyperpolarized MRI to assess intracellular $\mathrm{pH}$ in the in vivo heart has been demonstrated [39॰]. As mentioned previously, the ${ }^{13} \mathrm{CO}_{2}$ produced when $\left[1-{ }^{13} \mathrm{C}\right]$ pyruvate is decarboxylated by $\mathrm{PDH}$ is in a rapid, $\mathrm{pH}$ dependant equilibrium with bicarbonate $\left(\mathrm{H}^{13} \mathrm{CO}_{3-}\right)$. By measuring the relative intensities of the ${ }^{13} \mathrm{CO}_{2}$ and 
Fig. 1 An illustration of the metabolic pathways that have been examined with hyperpolarized MRI using the dynamic nuclear polarization (DNP) technique. AAT, alanine aminotransferase; CA, carbonic anhydrase; CAT, carnitine acetyltransferase; FM, fumarase; GLM, glutaminase; LDH, lactate dehydrogenase

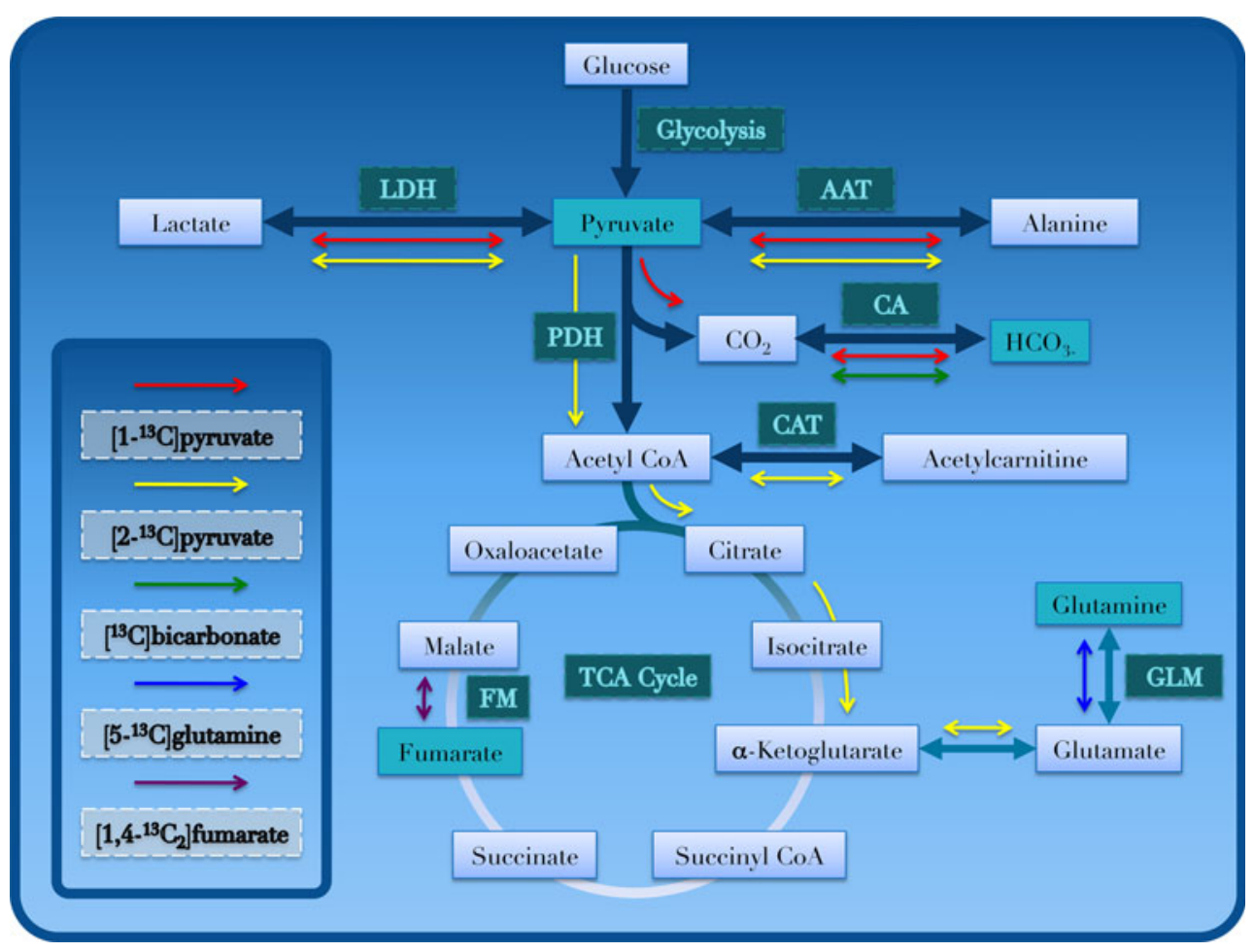

$\mathrm{H}^{13} \mathrm{CO}_{3}$ - resonances, it is possible to calculate the intracellular $\mathrm{pH}$ using the Henderson-Hasselbalch equation [39•].

Other metabolic pathways have been monitored in the heart. The use of $\left[2{ }^{13} \mathrm{C}\right]$ pyruvate has been shown to provide a method for the real-time assessment of metabolism through the TCA cycle (Fig. 1) [38••]. The use of pyruvate that is labeled with ${ }^{13} \mathrm{C}$ at the second carbon position means that the ${ }^{13} \mathrm{C}$ molecule is no longer cleaved into ${ }^{13} \mathrm{CO}_{2}$ but is carried into acetyl-CoA instead, allowing assessment of flux into the TCA cycle. In this work, Schroeder et al. demonstrated the ability to visualize the conversion of $\left[2-{ }^{13} \mathrm{C}\right]$ pyruvate into the downstream metabolites citrate, glutamate, and acetylcarnitine, and further, that the flux into the TCA cycle was reduced following a period of global ischemia.

One of the key issues to consider with the application of metabolic imaging using the DNP technique is that the molecules being injected are, by their very nature, metabolically active. They are also generally injected at supraphysiological concentrations. For example, in vivo experiments with $\left[1-{ }^{13} \mathrm{C}\right]$ pyruvate have frequently been carried out with injections of $\sim 80 \mathrm{mM}$ pyruvate, when the normal physiological concentration is $\sim 60 \mu \mathrm{M}$ [36]. As such, hyperpolarized compounds generated by DNP cannot be considered as "tracers" in the strictest sense of the word and the physiological consequences of the injected compound need to be considered [36, 40, 41]. To this aim, recent work has demonstrated that, due to saturation of either the mitochondrial pyruvate transporter or PDH itself, lower concentrations of pyruvate can be injected without affecting the level of bicarbonate signal observed [41, 42••]. Atherton et al. [36] have also shown that following a $1 \mathrm{~mL}$ injection of $80 \mathrm{mM}$ pyruvate into the rat, the maximum plasma concentration reached is only $250 \mu \mathrm{M}$. This relatively low concentration of pyruvate in the plasma, due most likely to the small volume injected and the rapid systemic uptake of pyruvate, is in line with pyruvate levels seen during exercise.

All of the work discussed so far on metabolic assessment with DNP has involved the use of unlocalized or "global" MR spectroscopy techniques. However, to visualize metabolic heterogeneity in the diseased heart, imaging techniques are required to distinguish healthy myocardium from diseased myocardium. As an initial approach to this, Golman et al. [9・•] demonstrated the use of a single slice, chemical shift imaging (CSI) acquisition scheme to allow the spatial localization of pyruvate, lactate, alanine, and bicarbonate following an injection of $\left[1-{ }^{13} \mathrm{C}\right]$ pyruvate into the pig heart. They further demonstrated localized alterations in the bicarbonate image following a period of ischemia caused by temporary occlusion of the left circumflex artery. The spatial resolution in this study was $7.5 \times 7.5 \times 20 \mathrm{~mm}$ and the total acquisition time was $13.4 \mathrm{~s}$ (non-ECG gated acquisition). This resolution has since been surpassed by Lau et al. [42••], who demonstrated a cardiac and respiratory gated, single-shot spiral acquisition technique with a spatial resolution of $3.7 \times 3.7 \times 10 \mathrm{~mm}$ and the ability to image any specific metabolite in $\sim 80 \mathrm{~ms}$. With this technique, whole heart coverage (6 slices) can be achieved within 3 heartbeats. 


\section{Conclusions}

The significant increases in MR signal that can be achieved with hyperpolarization techniques offer real promise in overcoming the sensitivity limitations of certain MRI applications. Dissolution DNP, and to a lesser extent PHIP, appear set to provide clinically relevant information in the cardiovascular applications of angiography, perfusion, catheter tracking, and metabolic imaging. However, neither DNP nor PHIP techniques have yet been transferred into humans and, until this milestone is achieved, their true clinical potential remains to be proven. In the case of DNP, this achievement appears to be imminent, with the first trials in man scheduled for late 2010 at the University of San Francisco in the study of prostate cancer. The conversion of DNP hyperpolarized $\left[1-{ }^{13} \mathrm{C}\right]$ pyruvate into $\left[1-{ }^{13} \mathrm{C}\right]$ lactate has been demonstrated to be a sensitive marker for tumor diagnosis and response to treatment $[7,8]$ and, therefore, the application of DNP in the field of oncology is generating a great deal of interest.

GE Healthcare has pioneered the development of a DNP polarizer that is capable of producing clinical-quality tracers, and the ability to polarize DNP tracers in sufficient quantities for human application has also been demonstrated [43]. Further work is still required to optimize the DNP technique for clinical applications, but significant steps have been taken in terms of increasing the polarization levels that can be achieved [44, 45], improving the MR pulse sequences used [46, 47], and improving the detection of hyperpolarized signals with new RF coils. The other significant area of current research has been the development of new tracer molecules to assess different metabolic pathways (Fig. 1). Hyperpolarized bicarbonate has been demonstrated for the measurement of extracellular $\mathrm{pH}$ [48], hyperpolarized glutamine has been demonstrated as a technique for assessing cellular proliferation [49], and hyperpolarized fumarate has been demonstrated as a marker of cellular necrosis [50].

The final translation of DNP into the clinical setting will undoubtedly present regulatory challenges that will need to be overcome. The delivery of any hyperpolarized tracer will require the removal of the electron radical before injection (a process which can be achieved by filtration of the dissolved tracer) and the impact of injecting supraphysiological concentrations of metabolically active compounds (as discussed above) will need to be carefully investigated. There will also be a need for rigorous and rapid quality control of the dissolved compounds in terms of the polarization level, $\mathrm{pH}$, temperature, etc. before injection into patients.

In summary, there is clear potential for hyperpolarized techniques, particularly DNP, to have a significant impact in the future of MRI. The cardiovascular applications that have been proposed look promising but require further development and greater validation against existing techniques. The potential for DNP in the area of metabolic imaging appears particularly strong. The ability to directly image the conversion of an injected compound into its downstream metabolites offers a distinct advantage over positron emission tomography, with the additional benefit of no ionizing radiation. There are still technical, practical, and regulatory hurdles to overcome but the gains in signal that can be achieved with hyperpolarization could provide unique tools to assess the heart.

Acknowledgments For their constant hard work, enthusiasm, and support, I would like to thank all of the members of the Cardiac Metabolism Research Group (CMRG) in Oxford, particularly Prof. Kieran Clarke, Prof. Sir George Radda, Dr. Marie Schroeder, Dr. Helen Atherton, Dr. Lowri Cochlin, Mr. Daniel Ball, and Mr. Michael Dodd. I would also like to acknowledge financial support from the British Heart Foundation, the Medical Research Council, and the Wellcome Trust and equipment support from GE Healthcare and Oxford Instruments Molecular Biotools.

Disclosure No potential conflict of interest relevant to this article was reported.

Open Access This article is distributed under the terms of the Creative Commons Attribution Noncommercial License which permits any noncommercial use, distribution, and reproduction in any medium, provided the original author(s) and source are credited.

\section{References}

Papers of particular interest, published recently have been highlighted as:

- Of importance

•- Of major importance

1. Mansson, S., E. Johansson, P. Magnusson, et al., $13 \mathrm{C}$ imaging- $a$ new diagnostic platform. Eur Radiol, 2006. 16(1): p. 57-67.

2. Bhattacharya, P., B.D. Ross and R. Bunger, Cardiovascular applications of hyperpolarized contrast media and metabolic tracers. Exp Biol Med (Maywood), 2009. 234(12): p. 1395-416.

3. Comment, A., B. van den Brandt, K. Uffmann, et al., Design and performance of a DNP prepolarizer coupled to a rodent MRI scanner. Concepts in Magnetic Resonance Part B-Magnetic Resonance Engineering, 2007. 31B(4): p. 255-269.

4. Fain, S.B., F.R. Korosec, J.H. Holmes, et al., Functional lung imaging using hyperpolarized gas MRI. J Magn Reson Imaging, 2007. 25(5): p. 910-23.

5. Viale, A., F. Reineri, D. Santelia, et al., Hyperpolarized agents for advanced MRI investigations. Q J Nucl Med Mol Imaging, 2009. 53(6): p. 604-17.

6. Ardenkjaer-Larsen, J.H., B. Fridlund, A. Gram, et al., Increase in signal-to-noise ratio of $>10,000$ times in liquid-state NMR. Proc Natl Acad Sci U S A, 2003. 100(18): p. 10158-63.

7. Day, S.E., M.I. Kettunen, F.A. Gallagher, et al., Detecting tumor response to treatment using hyperpolarized $13 \mathrm{C}$ magnetic resonance imaging and spectroscopy. Nat Med, 2007. 13(11): p. 1382-7. 
8. Golman, K., R.I. Zandt, M. Lerche, et al., Metabolic imaging by hyperpolarized $13 \mathrm{C}$ magnetic resonance imaging for in vivo tumor diagnosis. Cancer Res, 2006. 66(22): p. 10855-60.

9. •- Golman, K., J.S. Petersson, P. Magnusson, et al., Cardiac metabolism measured noninvasively by hyperpolarized 13C MRI. Magn Reson Med, 2008. 59(5): p. 1005-13. This article demonstrates the potential for hyperpolarized MRI to image the regional distribution of different metabolic products in normal and post-ischemic hearts.

10. • Merritt, M.E., C. Harrison, C. Storey, et al., Hyperpolarized 13C allows a direct measure of flux through a single enzyme-catalyzed step by NMR. Proc Natl Acad Sci U S A, 2007. 104(50): p. 19773-7. This article demonstrates the utility of hyperpolarized pyruvate in the perfused heart and shows that altered PDH flux can be assessed with high temporal resolution.

11. .- Schroeder, M.A., L.E. Cochlin, L.C. Heather, et al., In vivo assessment of pyruvate dehydrogenase flux in the heart using hyperpolarized carbon-13 magnetic resonance. Proc Natl Acad Sci U S A, 2008. 105(33): p. 12051-6. This is the first manuscript to use hyperpolarized pyruvate to assess the in vivo rat heart and to demonstrate the alterations to in vivo PDH flux caused by overnight fasting and type 1 diabetes.

12. - Golman, K. and J.S. Petersson, Metabolic imaging and other applications of hyperpolarized 13C1. Acad Radiol, 2006. 13(8): p. 932-42. This is an excellent review of the preliminary work exploring the potential applications of hyperpolarized MRI.

13. Ross, B.D., P. Bhattacharya, S. Wagner, et al., Hyperpolarized MR imaging: neurologic applications of hyperpolarized metabolism. AJNR Am J Neuroradiol, 2010. 31(1): p. 24-33.

14. Golman, K., J.H. Ardenkjaer-Larsen, J.S. Petersson, et al., Molecular imaging with endogenous substances. Proc Natl Acad Sci U S A, 2003. 100(18): p. 10435-9.

15. Golman, K., O. Axelsson, H. Johannesson, et al., Parahydrogeninduced polarization in imaging: subsecond (13)C angiography. Magn Reson Med, 2001. 46(1): p. 1-5.

16. Svensson, J., S. Mansson, E. Johansson, et al., Hyperpolarized 13C MR angiography using trueFISP. Magn Reson Med, 2003. 50(2): p. 256-62.

17. Olsson, L.E., C.M. Chai, O. Axelsson, et al., MR coronary angiography in pigs with intraarterial injections of a hyperpolarized 13C substance. Magn Reson Med, 2006. 55(4): p. 731-7.

18. Ishii, M., K. Emami, S. Kadlecek, et al., Hyperpolarized 13C MRI of the pulmonary vasculature and parenchyma. Magn Reson Med, 2007. 57(3): p. 459-63.

19. Schwitter, J., Myocardial perfusion imaging by cardiac magnetic resonance. J Nucl Cardiol, 2006. 13(6): p. 841-54.

20. Johansson, E., L.E. Olsson, S. Mansson, et al., Perfusion assessment with bolus differentiation: a technique applicable to hyperpolarized tracers. Magn Reson Med, 2004. 52(5): p. 1043-51.

21. Johansson, E., S. Mansson, R. Wirestam, et al., Cerebral perfusion assessment by bolus tracking using hyperpolarized 13C. Magn Reson Med, 2004. 51(3): p. 464-72.

22. Magnusson, P., E. Johansson, S. Mansson, et al., Passive catheter tracking during interventional MRI using hyperpolarized $13 C$. Magn Reson Med, 2007. 57(6): p. 1140-7.

23. Horowitz, J.D. and J.A. Kennedy, Time to address the cardiac metabolic "triple whammy" ischemic heart failure in diabetic patients. J Am Coll Cardiol, 2006. 48(11): p. 2232-4.

24. Neubauer, S., The failing heart-an engine out of fuel. N Engl J Med, 2007. 356(11): p. 1140-51.

25. Stanley, W.C., F.A. Recchia and G.D. Lopaschuk, Myocardial substrate metabolism in the normal and failing heart. Physiol Rev, 2005. 85(3): p. 1093-129.

26. Bailey, I.A., D.G. Gadian, P.M. Matthews, et al., Studies of metabolism in the isolated, perfused rat heart using $13 C$ NMR. FEBS Lett, 1981. 123(2): p. 315-8.
27. Lloyd, S., C. Brocks and J.C. Chatham, Differential modulation of glucose, lactate, and pyruvate oxidation by insulin and dichloroacetate in the rat heart. Am J Physiol Heart Circ Physiol, 2003. 285(1): p. H163-72.

28. Sherry, A.D., C.R. Malloy, P. Zhao, et al., Alterations in substrate utilization in the reperfused myocardium: a direct analysis by $13 \mathrm{C}$ NMR. Biochemistry, 1992. 31(20): p. 4833-7.

29. Chatham, J.C. and A.M. Seymour, Cardiac carbohydrate metabolism in Zucker diabetic fatty rats. Cardiovasc Res, 2002. 55(1): p. 104-12.

30. Malloy, C.R., A.D. Sherry and F.M. Jeffrey, Carbon flux through citric acid cycle pathways in perfused heart by $13 C$ NMR spectroscopy. FEBS Lett, 1987. 212(1): p. 58-62.

31. Ziegler, A., C.E. Zaugg, P.T. Buser, et al., Non-invasive measurements of myocardial carbon metabolism using in vivo $13 C$ NMR spectroscopy. NMR Biomed, 2002. 15(3): p. 222-34.

32. Malloy, C.R., J.R. Thompson, F.M. Jeffrey, et al., Contribution of exogenous substrates to acetyl coenzyme A: measurement by $13 C$ NMR under non-steady-state conditions. Biochemistry, 1990. 29 (29): p. 6756-61.

33. Kettunen, M.I., D.E. Hu, T.H. Witney, et al., Magnetization transfer measurements of exchange between hyperpolarized [113C]pyruvate and [1-13C]lactate in a murine lymphoma. Magn Reson Med, 2010. 63(4): p. 872-80.

34. Randle, P.J., Regulatory interactions between lipids and carbohydrates: the glucose fatty acid cycle after 35 years. Diabetes Metab Rev, 1998. 14(4): p. 263-83.

35. Tyler, D.J., M.A. Schroeder, L.E. Cochlin, et al., Application of hyperpolarized magnetic resonance in the study of cardiac metabolism. Applied Magnetic Resonance, 2008. 34(3-4): p. $523-531$.

36. Atherton, H.J., M.A. Schroeder, M.S. Dodd, et al., Validation of the in vivo assessment of pyruvate dehydrogenase activity using hyperpolarised (13)C MRS. NMR Biomed, 2010.

37. Merritt, M.E., C. Harrison, C. Storey, et al., Inhibition of carbohydrate oxidation during the first minute of reperfusion after brief ischemia: NMR detection of hyperpolarized 13CO2 and H13CO3. Magn Reson Med, 2008. 60(5): p. 1029-36.

38. •- Schroeder, M.A., H.J. Atherton, D.R. Ball, et al., Real-time assessment of Krebs cycle metabolism using hyperpolarized $13 \mathrm{C}$ magnetic resonance spectroscopy. FASEB J, 2009. 23(8): p. 2529-38. This article demonstrates the first application of $\left[2-{ }^{13} \mathrm{C}\right]$ pyruvate for the assessment of real-time flux through the TCA cycle and the alterations to this flux that are seen following global ischemia in the perfused heart.

39. - Schroeder, M.A., P. Swietach, H.J. Atherton, et al., Measuring intracellular $\mathrm{pH}$ in the heart using hyperpolarized carbon dioxide and bicarbonate: a $13 \mathrm{C}$ and $31 \mathrm{P}$ magnetic resonance spectroscopy study. Cardiovasc Res, 2010. 86(1): p. 82-91. This article details the in vivo utilization of $\left[1-{ }^{13}\right.$ C]pyruvate for the assessment of intracellular $p H$ in the heart with high temporal resolution.

40. Moreno, K.X., S.M. Sabelhaus, M.E. Merritt, et al., Competition of pyruvate with physiological substrates for oxidation by the heart: implications for studies with hyperpolarized [1-13C]pyruvate. Am J Physiol Heart Circ Physiol, 2010. 298(5): p. H1556-64.

41. Schroeder, M.A., H.J. Atherton, L.E. Cochlin, et al., The effect of hyperpolarized tracer concentration on myocardial uptake and metabolism. Magn Reson Med, 2009. 61(5): p. 1007-14.

42. •- Lau, A.Z., A.P. Chen, N.R. Ghugre, et al., Rapid multislice imaging of hyperpolarized (13)C pyruvate and bicarbonate in the heart. Magn Reson Med, 2010. A new acquisition scheme is presented which allows for rapid, cardiac-gated acquisition of metabolic images with high spatial and temporal resolution.

43. Comment, A., J. Rentsch, F. Kurdzesau, et al., Producing over $100 \mathrm{ml}$ of highly concentrated hyperpolarized solution by means of dissolution DNP. J Magn Reson, 2008. 194(1): p. 152-5. 
44. Jannin, S., A. Comment, F. Kurdzesau, et al., A $140 \mathrm{GHz}$ prepolarizer for dissolution dynamic nuclear polarization. J Chem Phys, 2008. 128(24): p. 241102.

45. Johannesson, H., S. Macholl and J.H. Ardenkjaer-Larsen, Dynamic Nuclear Polarization of [1-13C]pyruvic acid at 4.6 tesla. J Magn Reson, 2009. 197(2): p. 167-75.

46. Cunningham, C.H., A.P. Chen, M.J. Albers, et al., Double spinecho sequence for rapid spectroscopic imaging of hyperpolarized 13C. J Magn Reson, 2007. 187(2): p. 357-62.

47. Cunningham, C.H., A.P. Chen, M. Lustig, et al., Pulse sequence for dynamic volumetric imaging of hyperpolarized metabolic products. J Magn Reson, 2008. 193(1): p. 139-46.
48. Gallagher, F.A., M.I. Kettunen, S.E. Day, et al., Magnetic resonance imaging of $\mathrm{pH}$ in vivo using hyperpolarized $13 \mathrm{C}$ labelled bicarbonate. Nature, 2008. 453(7197): p. 940-3.

49. Gallagher, F.A., M.I. Kettunen, S.E. Day, et al., $13 C M R$ spectroscopy measurements of glutaminase activity in human hepatocellular carcinoma cells using hyperpolarized 13Clabeled glutamine. Magn Reson Med, 2008. 60(2): p. 2537.

50. Gallagher, F.A., M.I. Kettunen, D.E. Hu, et al., Production of hyperpolarized [1,4-13C2] malate from [1,4-13C2]fumarate is a marker of cell necrosis and treatment response in tumors. Proc Natl Acad Sci U S A, 2009. 106(47): p. 19801-6. 